\title{
Une conférence internationale sur l'ataxie de Friedreich
}

Le principal centre de recherche australien sur l'ataxie de Friedreich, le Murdoch Institute à Melbourne, organisait une conférence d'une journée pour faire le point des recherches sur la frataxine, sa fonction, et sur les stratégies thérapeutiques pour corriger son anomalie responsable de la maladie, l'expansion de triplets GAA [1].

Massimo Pandolfo (McGill, Montréal, Canada) a rappelé que les études menées chez la levure démontrent une accumulation de fer au niveau des complexes enzymatiques de la chaîne respiratoire mitochondriale en absence de frataxine $(\mathrm{m} / \mathrm{s}$ 1997, $n^{\circ} 10$, p. 1216). Le mécanisme d'action de la protéine n'est pas démontré, mais il serait d'exporter le fer hors de la mitochondrie. L'accumulation induirait une toxicité par augmentation de la production de radicaux libres. Les études effectuées chez l'homme confirment la localisation mitochondriale de la frataxine et la diminution considérable de son expression chez les malades $(\mathrm{m} / \mathrm{s}$ $\left.1998, n^{\circ} 1, p .104\right)$. Cela est toutefois spécifique des cellules des tissus cibles de la maladie, système nerveux, cœur et pancréas, car aucune anomalie du fer n'est détectée dans les fibroblastes à partir de biopsies de peau. Il existe également un déficit marqué des enzymes à fer et soufre du tissu cardiaque, sans anomalie au niveau des muscles squelettiques. Ces enzymes seraient particulièrement sensibles aux radicaux libres $(\mathrm{m} / \mathrm{s}$ 1998, $n^{\circ} 1, p$. 104). En revanche, l'ADN mitochondrial n'est pas atteint, quel que soit le tissu. Michel Koenig (Inserm U. 164, Strasbourg, France) a ensuite montré que la maladie était caucasienne; son âge de début et sa sévérité sont, comme dans la plupart des maladies dues à des expansions de triplets, corrélés à la taille de l'expansion [3], ellemême responsable d'une diminution, mais pas d'une disparition complète, de la protéine. Un bilan a été fait des cas où la maladie est liée à une mutation ponctuelle de la frataxine (moins de $5 \%$ ) et des indications fonctionnelles qui peuvent en être déduites à partir de la corrélation entre la mutation et le degré de sévérité de la maladie. En fait, et de façon surprenante, il existe également de très longues expansions muettes sur le plan clinique. L'avancement actuel des travaux sur des souris dont le gène de la frataxine a été délété ou exprimant une expansion du triplet GAA, permettront d'établir prochainement des modèles animaux de la maladie.

Des Richardson (Queensland, Melbourne, Australie) a présenté des travaux sur l'incorporation du fer dans l'hème dans la mitochondrie, avant son exportation vers le cytosol. Un inhibiteur, la succinylacétone, prévient l'incorporation du fer dans l'hème, et provoque une accumulation de fer.

Au plan thérapeutique, nous en sommes aux prémisses, sans espoir d'un tranfert rapide vers la clinique. Les traitements antioxydants, vitamine E par exemple, sont logiques et sans risques, mais leurs effets restent très limités. Erika Becker (Queensland, Melbourne, Australie) a fait le point des recherches sur les chélateurs du fer, le problème des effets secondaires qu'ils induisent, en particulier le déficit global en fer et l'impossibilité de les administrer par voie orale. Le chélateur de référence est la desferrioxamine, et le modèle d'étude privilégié analyse la capacité de chélation à partir de mitochondries surchargées en fer ou de globules rouges. Mais comment suivre les effets d'un traitement lors des futurs essais cliniques? Deux pistes sont explorées, celle de la résonnance magnétique nucléaire en spectroscopie, et l'utilisation de protéines liant le fer comme marqueurs biochimiques tels que la protoporphyrine IX et le malonyl aldéhyde plasmatique.

Beaucoup de progrès grâce aux connaissances fondamentales récemment acquises, beaucoup à faire pour arriver au traitement.

H.C.

1. Koenig M, Campuzano V, Cossée M, Mandel J. Ataxie de Friedreich : les expansions de triplets frappent encore. Med Sci 1996; 12: 431-5.

2. Mandel J. Maladies monogéniques et dysfonctions du système nerveux : progrès et perspectives. Med Sci 1996; 12 (suppl n $\left.{ }^{\circ} 10\right)$ : 100-8.

3. Imbert G, Saudou F, Yvert G, Mandel J, Cancel G, Brice A. Maladies neurodégénératives par expansion de polyglutamines: le sixième gène cloné (SCA2). Med Sci 1996; 12: 1463.

Société de Biologie 16 décembre 1998

Méiose : appariements et recombinaisons

Institut des Cordeliers

Amphithéâtre Bilski-Pasquier

15-21, rue de l'École-de-Médecine 75006 Paris, France

Renseignements

Secrétariat de la Société de Biologie 3 , rue d'Ulm, 75231 Paris Cedex 05, France

Tél./Fax : 0144271340 